\title{
Shifting Profile of MDR-TB Patients in Northern Portugal?
}

\author{
Olena Oliveira $^{1^{*}}$, A. Rita Gaio ${ }^{2,3}$, Anabela Silva ${ }^{4,5}$, Rita Macedo ${ }^{4}$ and Raquel Duarte ${ }^{1,4,6,7}$ \\ ${ }^{1}$ EPIUnit, Institute of Public Health, University of Porto, Portugal \\ ${ }^{2}$ Mathematics Department, Faculty of Sciences of the University of Porto, Porto, Portugal \\ ${ }^{3}$ Centre of Mathematics of the University of Porto, Porto, Portugal \\ ${ }^{4}$ Reference Centre for Multidrug-resistant tuberculosis in the North Region of Portugal, Chest Disease Centre of Vila Nova de Gaia, Portugal \\ ${ }^{5}$ National Health Institute Doutor Ricardo Jorge, Portugal \\ ${ }^{6}$ Pulmonology Department, Centro Hospitalar de Vila Nova de Gaia/Espinho, EPE, Vila Nova de Gaia, Portugal \\ ${ }^{7}$ Department of Clinical Epidemiology, Predictive Medicine and Public Health, Universityof Porto, Porto, Portugal
}

The occurrence of multidrug and extensively drug-resistant tuberculosis (M/XDR-TB), defined as a public health crisis by the World Health Organization (WHO) in 2013 [1], is a threat to all efforts to control TB in Europe. Despite the steady decline of TB incidence, Europe has faced the largest proportion of M/XDR-TB among individuals diagnosed with TB; this requires a stronger link between health system strengthening and M/XDR-TB control to adequately prevent and treat this deadly disease [2]. In 2014, $15 \%$ of new TB cases and $48 \%$ of previously treated cases were estimated to be MDR-TB [3].The XDR-TB prevalence among MDR-TB patients was $17.5 \%$ [4]. Within Europe the epidemiological pattern of TB varies greatly, with some countries showing a steady progress towards elimination of the disease, while others continue to face high rates of incidence [5].

Portugal is the only Western European country presenting an intermediate TB incidence, estimated at 20 per 100000 inhabitants in 2014, with the infection being concentrated at the two largest cities of Porto (Northern Region) and Lisbon (Lisbon and Tagus Valley Region) [6]. Nation wide, MDR-TB was reported for $2.5 \%$ of the cases with drug-susceptibility testing (DST) results, $26 \%$ of which XDR. MDR-TB was substantially circumscribed to the metropolitan areas of Porto and Lisbon [5]. In the Northern Region of Portugal, MDRTB was reported for $1.3 \%$ of the cases with DST results without any diagnosed cases of XDR-TB [7].

In 2007, the National TB Program defined specific strategies to control MDR-TB, based on the WHO Global Plan to Stop TB (20062015) [8]. The National Reference Centre for M/XDR-TB was created in June 2007 [9]. Facing the need to decentralize the approach of M/ XDR TB cases at a regional level, the Health National Directorate proposed the creation of 7 Regional Reference Centres for MDR-TB (CRRMR), one for each of the 7 Health Regions of the country.

The Northern Regional Reference Centre (NRRC) was the first unit starting its activity, in July 2009 [10].The NRRC is responsible for the clinical management of MDR-TB patients, including the choice and adjustment of treatment regimen according to the adverse effects, and the determination of need for hospitalization. Together with the family physician and public health authorities, the NRRC identifies the best strategy for contact tracing. Directly observed therapy is provided through the whole treatment.Simultaneously, the NRRC works in cooperation with community partners (such asdrug user's support centres, shelters and street teams) in the management of MDR-TB patients with riskbehaviours [11].

In the Northern Region of Portugal, in addition to the clinical TB notification from the National Tuberculosis Vigilance System (SVIG-TB), the notification of MDR-TB cases is complemented throughlaboratoryand pharmaceuticalnotification. Whenamultidrugresistant Mycobacterium tuberculosis strain is detected, the laboratory

\section{Publication History:}

Received: April 05, 2017

Accepted: June 28, 2017

Published: June 30, 2017

\section{Keywords:}

Tuberculosis, Multidrug, Treatment, Patients, Drug-susceptibility

notifies the reference centre and the clinician responsible for the patient; the pharmaceutical department notifies the reference centre the request of the second line drugsin healthcare services.

Our study describes and analyses trends and characteristics of M/XDR-TB patients diagnosed in the Northern Region of Portugal during 2003-2014.Throughout, we will then refer exclusively to this geographical area.Data were collected from SVIG-TB. We compared demographic (e.g., age, sex and country of origin) and clinical (e.g., prevalence of HIV, duration of treatment, the occurrence of adverse events and treatment outcome, etc.) characteristics in the period before (2003-2008) and after (2009-2014) the foundation of the NRRC.The treatment outcome was analysed for patients who have initiated treatment between 2003 and 2013(due to the recommended treatment duration - at least 18 months after culture conversion) [12]. The WHO standard definitions were used for both MDR/XDR-TB and treatment outcome(treatment success, died, failed, defaulted and transferred out)[12]. Toxicity during treatment was defined asany serious adverse event involving alteration of the treatment regimen.

During 2003-2014, 85 cases of MDR-TB were diagnosed. Table 1 shows the demographic and clinical characteristics of enrolled patients. The median age of the patients was 42 years-old (IQR 17),58 (68\%) patients were male, and 18 (21\%) were HIV positive. The median time of treatment was 19 months and 53 (71\%) patients successfully completed treatment.

Although thedifferences were not statistically significant, coinfection with HIV (25\% vs $14 \%)$, alcohol abuse $(40 \%$ vs $32 \%)$ and injection drug use (27 vs $12 \%$ ) were more prevalent in the first period. Four cases of XDR-TB were diagnosed in this period. The proportion of foreign-born was more prevalent in the second period $(22 \%$ vs

"Corresponding Author: Dr. Olena Oliveira, EPIUnit, Institute of Public Health University of Porto, Portugal; E-mail: abernardo@egasmoniz.edu.pt

Citation: Oliveira O, Gaio AR, Silva A, Macedo R, Duarte R (2017) Shifting Profile of MDR-TB Patients in Northern Portugal?. Int J Clin Res Trials 2: 114. doi: https://doi.org/10.15344/2456-8007/2017/114

Copyright: (c) 2017 Oliveira et al. This is an open-access article distributed under the terms of the Creative Commons Attribution License, which permits unrestricted use, distribution, and reproduction in any medium, provided the original author and source are credited. 
Citation: Oliveira O, Gaio AR, Silva A, Macedo R, Duarte R (2017) Shifting Profile of MDR-TB Patients in Northern Portugal?. Int J Clin Res Trials 2: 114. doi: https://doi.org/10.15344/2456-8007/2017/114

Page 2 of 2

10\%). The prevalence of males and previously treated TB patients was similar in both periods. The treatment success rate was $66 \%$ and $77 \%$ in the first and second periods, respectively.

In the present study, between 2003 and 2014, a decreasing trend of MDR-TB was observed. This decrease followed the decline of TB in the region, maintaining its proportion at $0.6 \%$. This decrease is consistent with the decrease observed at the national level [6]. Comparing the patients' characteristics in the periods of 2003-2008 and 2009-2014, the patient profile seems to be different within each period, although no significant differences have been identified. A higher prevalence of patients with risk behaviour (alcohol abuse and injection drug) and of younger age is observed within the first period. The prevalence of HIV among MDR-TB patients has also decreased (from 27\% to 14\%) reflecting the decrease of the HIV incidence and TB/HIV co-infection in Portugal since 2003 [6]. The prevalence of HIV positive TB patients in the period $2003-2014$ decreased from $27 \%$ to $12 \%$ [6].

In our study, the proportion of foreign-born MDR-TB patients in the Northern region was higher in the second period. Nationwide, in this period, a similar trend was observed in MDR-TB, but not in susceptible TB [6]. According to the $\mathrm{WHO}$ guidelines, the recommended treatment duration is $18-24$ months, determined by culture conversion and disease extension [12]. This recommendation was accomplished in the second period (20 months). It was also during this period that the occurrence of toxicity during treatment was lower (4\%). The goal of The Global Plan was to achieve a treatment success rate of at least $75 \%$ in MDR-TB patients by 2015 [13]. In Europe and in Portugal, in the 2012 cohort, the treatment success rate was $40 \%$ [4] and 77\% respectively [5]. Our data showed that the treatment success rate hasincreased from $66 \%$ (2003-2008) to $77 \%$ (2009-2013). However, we found no association between the factors analysed andasuccessful treatment.

The strength of this study is the completeness of ourdata that, through the triple notification system,allows forthe identification of all MDR-TB cases while the limitations consist of the limited number of factors assessedand lack of statistical power (small number of cases analysed).

In conclusion, in the Northern Region of Portugal, the centralization of MDRTB cases in one expert centre was followed by an improvement of the MDR-TB case management and treatment outcome. We seem to beattending a shift in the patients' profile, though yet not statistically significant. Patients areno longer essentially young natives with HIV and drug abuse but older and foreigner individuals instead.

\section{Competing Interests}

The authors declare that they have no competing interests.

\section{Author Contributions}

Study conception and design: O. Oliveira, R. Duarte

Analysis and interpretation of data: O. Oliveira, A.R. Gaio, R. Duarte

Drafting of manuscript: O. Oliveira, R. Duarte

Critical revision: A.R. Gaio, A. Silva, R. Macedo, R. Duarte

\section{References}

1. World Health Organization. Reaching people with MDR-TB. Progressing in diagnosis: a key step in overcoming the MDR-TB crisis. Geneve, WHO, 2014.
2. World Health Organization. Best practices in prevention, control and care for drug- resistant tuberculosis. Copenhagen, WHO Regional Office for Europe, 2013.

3. World Health Organization. Global tuberculosis report 2015. WHO/HTM/ TB/2015.22 Geneva, WHO, 2014.

4. European Centre for Disease Prevention and Control (2016) Annual Epidemiological Report, 2016 - Tuberculosis. Stockholm: ECDC.

5. European Centre for Disease Prevention and Control. ECDC special report. Progressing towards TB elimination. A follow-up to the Framework Action Plan to Fight Tuberculosis in the European Union. Stockholm: ECDC; 2016.

6. Direção - Geral da Saúde. Portugal, Infeção VIH/SIDA e Tuberculose emnúmeros-2015. Programa Nacional para a Infeção VIH/SIDA

7. Direção -Geral da Saúde. SVIG-TB.

8. Gomes C. CRTMR Programa Nacional de Luta Contra a Tuberculose. As recomendações para a gestão da TBMR. DGS/PNT/CRTMR/2008.001. Lisboa, DGS, 2008.

9. Direcção - Geral da Saúde. Centro de Referência para Tuberculose Multirresistente (CRTMR). Circular Informativa no 14/DT de 05/06/07. Lisboa: DGS, 2007.

10. Administração Regional de Saúde do Norte. Centro de Referencia Regional para a TBMR. ARS Norte, 23 de Abril 2009.

11. Duarte R, Santos A, Mota M, Carvalho A, Marques A, et al. (2011) Involving community partners in the management of tuberculosis among drug users. Public Health 125: 60-62.

12. World Health Organization. Guidelines for the programmatic management of drug-resistant tuberculosis. Emergency update, 2008. WHO/HTM/TB/2008.402. Geneva, WHO, 2008.

13. World Health Organization. The global plan to stop TB 2011-2015: transforming the fight towards elimination of tuberculosis. WHO/HTM/STB/2010.2. Geneva, WHO 2010. 